\title{
Monotone Iterative Solutions for Nonlinear Boundary Value Problems of Fractional Differential Equation
}

\author{
Chaozhu Hu, Bin Liu, and Songfa Xie \\ School of Mathematics and Statistics, Huazhong University of Science and Technology, Wuhan 430074, Hubei, China \\ Correspondence should be addressed to Bin Liu; binliu@mail.hust.edu.cn
}

Received 27 April 2013; Accepted 7 August 2013

Academic Editor: Yong Zhou

Copyright (C) 2013 Chaozhu Hu et al. This is an open access article distributed under the Creative Commons Attribution License, which permits unrestricted use, distribution, and reproduction in any medium, provided the original work is properly cited.

\begin{abstract}
By means of the method of quasi-lower and quasi-upper solutions and monotone iterative technique, we consider the nonlinear boundary value problems with Caputo fractional derivative and introduce two well-defined monotone sequences of quasi-lower and quasi-upper solutions which converge uniformly to the actual solution of the problem, and then the existence results of the solution for the problems are established. A numerical iterative scheme is introduced to obtain an accurate approximate solution and to give one example to demonstrate the accuracy and efficiency of the new approach.
\end{abstract}

\section{Introduction}

We are interested in the existence and uniqueness of solution of the following nonlinear boundary value problem of fractional order

$$
\begin{gathered}
D^{\mu} u(t)+F(t, u)=0, \quad t \in(0,1), \\
u(0)=\alpha, \quad u(1)=\beta,
\end{gathered}
$$

where $1<\mu \leq 2, F(t, u)=f_{0}(t, u)+f_{1}(t, u)+f_{2}(t, u)$, and $f_{i}:[0,1] \times R \rightarrow R(i=0,1,2)$ are a continuous function, $\alpha, \beta \in R$, and $D^{\mu}$ is the Caputo fractional derivative of order $\mu$.

It is well known that the differential equations with fractional order are generalization of ordinary differential equations to noninteger order, they occur more frequently in different research areas and engineering, such as physics, control of dynamical systems, chemistry and so forth. We also remark that several kinds of fractional derivatives were introduced to investigate the fractional differential equation, see, for example, $[1,2]$, and references therein.

Roughly speaking, it is a difficult task to give exact solutions for fractional differential equations. Recently, there are a number of numerical and analytical techniques to concerned with such problems, for instance, the homotopy analysis method, the Adomian decomposition method and the critical point theory have been discussed the fractional differential equations, such as [3-9]. On the other hand, very recently, the monotone iterative technique, combined with the method of lower and upper solutions were introduced to study the problems [10-13]. In [10, 11], the authors used the method of lower and upper solutions to investigate the existence of solutions for a class of fractional initial value problems. In [12] the authors considered fractional boundary value problem and proved the existence of solution. In [13], the author discussed the Monotone iterative technique for boundary value problems of a nonlinear fractional differential equation with deviating arguments. Specially, here it is worth mentioning, that Al-Refai and Ali Hajji [14] introduce two well-defined monotone sequences of lower and upper solutions which converge uniformly to the actual solution of the problem. However, the existence results in [14] mainly depend upon a restrictive condition; that is,

$$
F(t, u) \text { is strictly decreasing with respect to } u \text {. }
$$

It is a critical condition in order to discuss the monotone iterative sequences. It is, therefore, natural to ask how to discuss the problem (1), if $F(t, u)$ is not necessary decreasing with respect to $u$. So, being directly inspired by [14], the purpose of this paper is to study the nonlinear boundary value problems of fractional order with Dirichlet boundary conditions. We introduce a method based on quasi-lower and quasi-upper solutions to prove the existence of a unique 
solution with no claim on the strictwe monotone. And also give an algorithm to construct two monotone sequences of quasi-lower and quasi-upper solutions. Moreover, the constructed sequences are proved to converge uniformly to the unique solution of the problem.

The paper is organized as follows. Preliminaries are in Section 2. Then in Section 3, we construct the monotone sequences of quasi-lower and quasi-upper solutions and prove their uniform convergence to the unique solution of the problem. Finally, in Section 4, we establish the numerical approach employed to obtain accurate numerical solution, and give one example to demonstrate the accuracy and efficiency of the new approach.

\section{Preliminaries}

In this sections, we present the definition of a pair of quasilower and quasi-upper solutions and some lemmas which will be needed in the next section.

For $\theta>0, m-1<\theta \leq m, m \in N$, the Caputo derivative (see $[1,2])$ is defined by

$$
D^{\theta} h(t)=\frac{1}{\Gamma(m-\theta)} \int_{0}^{t}(t-s)^{m-1-\theta} h^{(m)}(s) d s .
$$

The Caputo derivative defined in (3) is related to the Riemann-Liuville fractional integral $I^{\theta}$ of $\theta \in R^{+}$by

$$
D^{\theta} h(t)=I^{m-\theta} h^{(m)}(t),
$$

where for any $\delta>0$

$$
I^{\delta} h(t)=\frac{1}{\Gamma(\delta)} \int_{0}^{t}(t-s)^{\delta-1} h(s) d s
$$

Moreover,

$$
\begin{gathered}
I^{\theta} D^{\theta} h(t)=h(t)-\sum_{k=0}^{m-1} c_{k} t^{k}, \\
D^{\theta} I^{\theta} h(t)=h(t),
\end{gathered}
$$

where $c_{k}=h^{(k)}\left(0^{+}\right) / k !, 1 \leq k \leq m-1$.

Definition 1. The functions $v^{(0)}, w^{(0)} \in C^{2}([0,1], R)$ are called a pair of quasi-lower and quasi-upper solutions of the problem (1), if it satisfies

$$
\begin{gathered}
D^{\mu} v^{(0)}(t)+f_{0}\left(t, v^{(0)}\right)+f_{1}\left(t, v^{(0)}\right)+f_{2}\left(t, w^{(0)}\right) \geq 0, \\
v^{(0)}(0) \leq \alpha, \quad v^{(0)}(1) \leq \beta, \\
D^{\mu} w^{(0)}(t)+f^{(0)}\left(t, w^{(0)}\right)+f_{1}\left(t, w^{(0)}\right)+f_{2}\left(t, v^{(0)}\right) \leq 0, \\
w^{(0)}(0) \geq \alpha, \quad w^{(0)}(1) \geq \beta .
\end{gathered}
$$

If the above inequalities are equalities, we call $v^{(0)}$ and $w^{(0)}$ a pair of quasi-solutions of the problem (1).
Lemma 2 (see [14]). Let $p \in C^{2}([0,1], R)$ and $C>0$ is a constant. If $p(t)$ satisfies the relations

$$
\begin{gathered}
D^{\mu} p(t)-C p(t) \leq 0, \quad t \in(0,1), \\
p(0), p(1) \geq 0,
\end{gathered}
$$

then $p(t) \geq 0$ for $t \in[0,1]$.

Lemma 3 (see [14]). A function $u(t)$ is a solution of the linear fractional BVP problems

$$
\begin{gathered}
D^{\mu} u(t)-C u(t)=h(t), \quad t \in(0,1), \\
u(0)=\alpha, \quad u(1)=\beta,
\end{gathered}
$$

if and only if it is a solution of integral equation

$$
u(t)=\alpha+(\beta-\alpha) t-\int_{0}^{1} G(s, t)(C u(s)+h(s)) d s,
$$

where

$$
G(s, t)=\frac{1}{\Gamma(\mu)} \begin{cases}t(1-s)^{\mu-1}-(t-s)^{\mu-1}, & 0 \leq s \leq t \leq 1 \\ t(1-s)^{\mu-1}, & 0 \leq t \leq s \leq 1\end{cases}
$$

Lemma 4. For all $t \in[0,1]$, we have

$$
0 \leq \int_{0}^{1} G(s, t) d s \leq \frac{1}{\Lambda \Gamma(\mu)}
$$

where

$$
\Lambda=\mu^{\mu /(\mu-1)} \cdot \frac{\mu}{\mu-1} .
$$

Proof. The proof of this lemma is easy, so we omit it.

In the rest of the paper, for convenience sake, we define the set

$$
[x, y]=\left\{z \in C^{2}([0,1], R): x(t) \leq z(t) \leq y(t), t \in[0,1]\right\},
$$

let

$$
\|x\|=\sup _{t \in[0,1]}|x(t)|
$$

and list some conditions.

$\left(C_{1}\right) v^{(0)}, w^{(0)}$ is a pair of quasi-lower and quasi-upper solutions of the problem (1) and $v^{(0)}(t) \leq w^{(0)}(t)$, $t \in[0,1]$.

$\left(C_{2}\right)$ The function $f_{1}(t, u)$ is increasing with respect to $u$, and $f_{2}$ is decreasing with respect to $u$. And there exists a constant $M \in(0, \Lambda \Gamma(\mu))$ such that

$$
f_{0}(t, u)-f_{0}(t, \bar{u}) \geq-M(u-\bar{u}), \quad \forall v^{(0)} \leq \bar{u} \leq u \leq w^{(0)}
$$


Lemma 5. Let $\left(C_{1}\right)$ and $\left(C_{2}\right)$ hold; then for any fixed $\eta_{1}, \eta_{2} \in$ $\left[v^{(0)}, w^{(0)}\right]$, the linear problems

$$
\begin{aligned}
D^{\mu} u(t)= & M\left(u(t)-\eta_{1}(t)\right)-f_{0}\left(t, \eta_{1}(t)\right) \\
& -f_{1}\left(t, \eta_{1}(t)\right)-f_{2}\left(t, \eta_{2}(t)\right), \quad t \in(0,1) \\
u(0)= & \alpha, \quad u(1)=\beta,
\end{aligned}
$$

have a unique solution $u_{\eta_{1}, \eta_{2}} \in\left[v^{(0)}, w^{(0)}\right], t \in[0,1]$.

Proof. Firstly, we prove that if $\bar{u}_{\eta_{1}, \eta_{2}}$ is a solution of (17), then $\bar{u}_{\eta_{1}, \eta_{2}} \in\left[v^{(0)}, w^{(0)}\right]$. From $\left(C_{1}\right),\left(C_{2}\right)$ and if $v^{(0)}$ is a quasi-lower solution of the problem (1), then we have

$$
\begin{aligned}
D^{\mu} v^{(0)}(t) \geq & -f_{0}\left(t, v^{(0)}\right)-f_{1}\left(t, v^{(0)}\right)-f_{2}\left(t, w^{(0)}\right) \\
\geq & M\left(v^{(0)}(t)-\eta_{1}(t)\right)-f_{0}\left(t, \eta_{1}(t)\right) \\
& -f_{1}\left(t, \eta_{1}(t)\right)-f_{2}\left(t, \eta_{2}(t)\right), \\
& v^{(0)}(0) \leq \alpha, \quad v^{(0)}(1) \leq \beta .
\end{aligned}
$$

Thus from (18) and if $\bar{u}_{\eta_{1}, \eta_{2}}$ is a solution of (17), set $p(t)=$ $\bar{u}_{\eta_{1}, \eta_{2}}(t)-v^{(0)}(t)$; then

$$
\begin{gathered}
D^{\mu} p(t)-M p(t) \leq 0, \quad t \in(0,1) \\
p(0), p(1) \geq 0 .
\end{gathered}
$$

By Lemma 2, we have that

$$
p(t) \geq 0, \quad t \in[0,1] .
$$

That is, $\bar{u}_{\eta_{1}, \eta_{2}} \geq v^{(0)}$. Similarly, we show $\bar{u}_{\eta_{1}, \eta_{2}} \leq w^{(0)}$. Therefore, $\bar{u}_{\eta_{1}, \eta_{2}} \in\left[v^{(0)}, w^{(0)}\right]$.

Next, we show that (17) has a unique solution.

From Lemma 3, the problems (17) are equivalent to the following integral equation

$$
u(t)=\alpha+(\beta-\alpha) t-\int_{0}^{1} G(s, t)\left(M u(s)-h_{\eta_{1}, \eta_{2}}(s)\right) d s,
$$

where $h_{\eta_{1}, \eta_{2}}(t)=f_{0}\left(t, \eta_{1}(t)\right)+f_{1}\left(t, \eta_{1}(t)\right)+f_{2}\left(t, \eta_{2}(t)\right)+$ $M \eta_{1}(t)$.

Let

$$
\begin{array}{r}
\mathscr{A} u(t)=\alpha+(\beta-\alpha) t-\int_{0}^{1} G(s, t)\left(M u(s)-h_{\eta_{1}, \eta_{2}}(s)\right) d s \\
t \in[0,1] .
\end{array}
$$

For any $u, \bar{u} \in C^{2}([0,1], R)$, by Lemma 4 and condition $\left(C_{2}\right)$, we have

$$
\begin{aligned}
|\mathscr{A} u(t)-\mathscr{A} \bar{u}(t)| & \leq M \int_{0}^{1} G(s, t)|u(s)-\bar{u}(s)| d s \\
& \leq M \int_{0}^{1} G(s, t) d s\|u-\bar{u}\| \\
& \leq \frac{M}{\Lambda \Gamma(\mu)}\|u-\bar{u}\|<\|u-\bar{u}\| .
\end{aligned}
$$

So

$$
\|\mathscr{A} u-\mathscr{A} \bar{u}\|<\|u-\bar{u}\| .
$$

By the Banach fixed point theorem, the operator $\mathscr{A}$ has a unique point. This shows that (17) has a unique solution $u \in$ $\left[v^{(0)}, w^{(0)}\right], t \in[0,1]$.

\section{Monotone Sequences of Quasi-Lower and Quasi-Upper Solutions}

In this sections we construct the monotone sequences of quasi-lower and quasi-upper solutions and prove their uniform convergence to the unique solution of the problem.

Theorem 6. Let $\left(C_{1}\right)$ and $\left(C_{2}\right)$ hold, and assume that $\left(C_{3}\right) v^{(k)}, w^{(k)}, k \geq 1$ is a pair of solutions of

$$
\begin{aligned}
D^{\mu} v^{(k)}= & M\left(v^{(k)}-M v^{(k-1)}\right)-f_{0}\left(t, v^{(k-1)}\right) \\
& -f_{1}\left(t, v^{(k-1)}\right)-f_{2}\left(t, w^{(k-1)}\right), \quad t \in(0,1), \\
& v^{(k)}(0)=\alpha, \quad v^{(k)}(1)=\beta, \\
D^{\mu} w^{(k)}= & M\left(w^{(k)}-M w^{(k-1)}\right)-f_{0}\left(t, w^{(k-1)}\right) \\
& -f_{1}\left(t, w^{(k-1)}\right)-f_{2}\left(t, v^{(k-1)}\right), \quad t \in(0,1), \\
& w^{(k)}(0)=\alpha, \quad w^{(k)}(1)=\beta .
\end{aligned}
$$

Then one has the following.

(i) The sequence $v^{(k)}, w^{(k)}$ is a pair of quasi-lower and quasi-upper solutions of (1). Moreover,

$$
v^{(0)} \leq v^{(1)} \leq \cdots \leq v^{(k)} \leq \cdots \leq w^{(k)} \leq \cdots \leq w^{(1)} \leq w^{(0)} .
$$

(ii) The sequence $v^{(k)}, w^{(k)}$ converges uniformly to $v_{*}$ and $w^{*}$, respectively, with $v^{(0)} \leq v_{*} \leq w^{*} \leq w^{(0)}$. Moreover, $v_{*}$ and $w^{*}$ are a pair of minimal-maximal quasi-solutions of (1) in $\left[v^{(0)}, w^{(0)}\right]$.

(iii) Suppose further that there exist constants $L_{i}>0(i=$ $0,1,2)$ such that $L_{1}+L_{2}<L_{0} \leq M$, and

$$
\begin{array}{r}
f_{0}(t, u)-f_{0}(t, \bar{u}) \leq-L_{0}(u-\bar{u}), \\
f_{1}(t, u)-f_{1}(t, \bar{u}) \leq L_{1}(u-\bar{u}), \\
\forall v^{(0)} \leq \bar{u} \leq u \leq w^{(0)}, \\
f_{2}(t, u)-f_{2}(t, \bar{u}) \geq-L_{2}(u-\bar{u}) .
\end{array}
$$

Then $v_{*}=w^{*}$ is the actual solution of (1) in $\left[v^{(0)}, w^{(0)}\right]$. 
Proof. (i) For any fixed $\eta_{1}, \eta_{2} \in\left[v^{(0)}, w^{(0)}\right]$, consider the linear problems

$$
\begin{gathered}
D^{\mu} u(t)=M\left(u(t)-\eta_{1}(t)\right)-f_{0}\left(t, \eta_{1}(t)\right) \\
-f_{1}\left(t, \eta_{1}(t)\right)-f_{2}\left(t, \eta_{2}(t)\right), \quad t \in(0,1) \\
u(0)=\alpha, \quad u(1)=\beta .
\end{gathered}
$$

By Lemma 5, the linear problems (28) have a unique solution $u_{\eta_{1}, \eta_{2}} \in\left[v^{(0)}, w^{(0)}\right]$. Define operator $\mathscr{B}:\left[v^{(0)}, w^{(0)}\right] \times$ $\left[v^{(0)}, w^{(0)}\right] \rightarrow\left[v^{(0)}, w^{(0)}\right]$ as follows:

$$
\mathscr{B}\left[\eta_{1}, \eta_{2}\right]=u_{\eta_{1}, \eta_{2}}
$$

Again, it is easy that to prove that $\eta_{1}, \eta_{2}$ are a pair of quasisolutions of the problem (1) if and only if

$$
\begin{aligned}
& \eta_{1}=\mathscr{B}\left[\eta_{1}, \eta_{2}\right], \\
& \eta_{2}=\mathscr{B}\left[\eta_{2}, \eta_{1}\right] .
\end{aligned}
$$

Furthermore, from (28) and condition $\left(C_{2}\right)$, we can prove $\mathscr{B}\left[\eta_{1}, \eta_{2}\right]$ is increasing with respect to $\eta_{1}$, and is decreasing with respect to $\eta_{2}$; that is,

$$
\begin{gathered}
\forall \eta, \eta_{1}, \eta_{2} \in\left[v^{(0)}, w^{(0)}\right], \\
\eta_{1} \leq \eta_{2} \Longrightarrow\left\{\begin{array}{l}
\mathscr{B}\left[\eta_{1}, \eta\right] \leq \mathscr{B}\left[\eta_{2}, \eta\right], \\
\mathscr{B}\left[\eta, \eta_{1}\right] \geq \mathscr{B}\left[\eta, \eta_{2}\right] .
\end{array}\right.
\end{gathered}
$$

In fact, setting $u_{1}=\mathscr{B}\left[\eta_{1}, \eta\right], u_{2}=\mathscr{B}\left[\eta_{2}, \eta\right]$, by (28) and condition $\left(C_{2}\right)$, we have

$$
\begin{aligned}
D^{\mu} u_{1} & (t)-M u_{1}(t) \\
= & -M \eta_{1}(t)-f_{0}\left(t, \eta_{1}(t)\right)-f_{1}\left(t, \eta_{1}(t)\right)-f_{2}(t, \eta(t)) \\
\geq & -M \eta_{2}(t)-f_{0}\left(t, \eta_{2}(t)\right)-f_{1}\left(t, \eta_{2}(t)\right)-f_{2}(t, \eta(t)) \\
= & D^{\mu} u_{2}(t)-M u_{2}(t), \\
& u_{1}(0)=\alpha=u_{2}(0), \quad u_{1}(1)=\beta=u_{2}(1) .
\end{aligned}
$$

Thus, by setting $p(t)=u_{2}(t)-u_{1}(t)$, then

$$
\begin{gathered}
D^{\mu} p(t)-M p(t) \leq 0, \\
p(0), p(1)=0 .
\end{gathered}
$$

By Lemma 2, we have that

$$
p(t) \geq 0, \quad t \in[0,1] \text {. }
$$

That is, $u_{2} \geq v_{1}$, or the first inequality holds in (31). Similarly, the second inequality holds in (31) too.

Again, similar to the previous argument, we can show that

$$
v^{(0)} \leq \mathscr{B}\left[v^{(0)}, w^{(0)}\right], \quad \mathscr{B}\left[w^{(0)}, v^{(0)}\right] \leq w^{(0)} .
$$

Now, let

$$
\begin{array}{r}
v^{(k)}=\mathscr{B}\left[v^{(k-1)}, w^{(k-1)}\right], \quad w^{(k)}=\mathscr{B}\left[w^{(k-1)}, v^{(k-1)}\right], \\
k=1,2, \ldots .
\end{array}
$$

Thus, from (31), (35), and (36), we have

$$
v^{(0)} \leq v^{(1)} \leq \cdots \leq v^{(k)} \leq \cdots \leq w^{(k)} \leq \cdots \leq w^{(1)} \leq w^{(0)} .
$$

Moreover, it is clear that $v^{(k)}, w^{(k)}$ satisfy (36) if and only if $v^{(k)}, w^{(k)}$ are a pair of solutions of (25).

To prove $(i)$, we only need to show that $v^{(k)}, w^{(k)}$ are a pair of quasi-lower and quasi-upper solutions of (1). In fact, from (25) and condition $\left(C_{2}\right)$, we can obtain

$$
\begin{aligned}
D^{\mu} v^{(k)}+ & f_{0}\left(t, v^{(k)}\right)+f_{1}\left(t, v^{(k)}\right)+f_{2}\left(t, w^{(k)}\right) \\
= & M\left(v^{(k)}-v^{(k-1)}\right)+f_{0}\left(t, v^{(k)}\right)-f_{0}\left(t, v^{(k-1)}\right) \\
& +f_{1}\left(t, v^{(k)}\right)-f_{1}\left(t, v^{(k-1)}\right) \\
& +f_{2}\left(t, w^{(k)}\right)-f_{2}\left(t, w^{(k-1)}\right) \geq 0, \\
& v^{(k)}(0)=\alpha, \quad v^{(k)}(1)=\beta, \\
D^{\mu} w^{(k)}+ & f_{0}\left(t, w^{(k)}\right)+f_{1}\left(t, w^{(k)}\right)+f_{2}\left(t, v^{(k)}\right) \\
= & M\left(w^{(k)}-w^{(k-1)}\right)+f_{0}\left(t, w^{(k)}\right)-f_{0}\left(t, w^{(k-1)}\right) \\
+ & f_{1}\left(t, w^{(k)}\right)-f_{1}\left(t, w^{(k-1)}\right) \\
+ & f_{2}\left(t, v^{(k)}\right)-f_{2}\left(t, v^{(k-1)}\right) \leq 0, \\
& v^{(k)}(0)=\alpha, \quad v^{(k)}(1)=\beta .
\end{aligned}
$$

Hence, $v^{(k)}, w^{(k)}$ is a pair of quasi-lower and quasi-upper solutions of (1). This proves (i).

(ii) Since the sequence $v^{(k)}$ is monotone nondecreasing and is bounded from above mention, the sequence $w^{(k)}$ is monotone nonincreasing and is bounded from below, therefore the pointwise limits exist and these limits are denoted by $v_{*}$ and $w^{*}$. The sequence $v^{(k)}, w^{(k)}$ are sequences of continuous functions defined on the compact set $[0,1]$, hence By Dini's theorem [15], the convergence is uniform. This is

$$
v^{(0)} \leq v_{*}=\lim _{k \rightarrow \infty} v^{(k)} \leq \lim _{k \rightarrow \infty} w^{(k)}=w^{*} \leq w^{(0)}
$$

uniformly on $t \in[0,1]$. 
Moveover, from (25), we have

$$
\begin{gathered}
D^{\mu} v_{*}=-f_{0}\left(t, v_{*}\right)-f_{1}\left(t, v_{*}\right)-f_{2}\left(t, w^{*}\right), \quad t \in(0,1), \\
v_{*}(0)=\alpha, \quad v_{*}(1)=\beta, \\
D^{\mu} w^{*}=-f_{0}\left(t, w^{*}\right)-f_{1}\left(t, w^{*}\right)-f_{2}\left(t, v_{*}\right), \quad t \in(0,1), \\
w^{*}(0)=\alpha, \quad w^{*}(1)=\beta .
\end{gathered}
$$

This show that $v_{*}, w^{*}$ are a pair of quasi-solutions of the problem (1).

Now, we prove that $v_{*}$ and $w^{*}$ are a pair of minimalmaximal quasi-solutions of (1).

Let $v, w \in\left[v^{(0)}, w^{(0)}\right]$ be a pair of quasi-solutions of the problem (1), in the following, we show this using induction arguments. In fact, we have

$$
v^{(0)} \leq v, \quad w \leq w^{(0)} .
$$

Assume that

$$
v^{(k-1)} \leq v, \quad w \leq w^{(k-1)}
$$

is true. Thus from (31) and (36), we can obtain

$$
\begin{aligned}
& v^{(k)}=\mathscr{B}\left[v^{(k-1)}, w^{(k-1)}\right] \leq \mathscr{B}[v, w]=v, \\
& v^{(k)}=\mathscr{B}\left[v^{(k-1)}, w^{(k-1)}\right] \leq \mathscr{B}[w, v]=w, \\
& w^{(k)}=\mathscr{B}\left[w^{(k-1)}, v^{(k-1)}\right] \geq \mathscr{B}[v, w]=v, \\
& w^{(k)}=\mathscr{B}\left[w^{(k-1)}, v^{(k-1)}\right] \geq \mathscr{B}[w, v]=w .
\end{aligned}
$$

Therefore,

$$
v^{(k)} \leq v, \quad w \leq w^{(k)}, \quad k=0,1,2, \ldots
$$

Thus, taking limit in (44) as $k \rightarrow \infty$, we have

$$
v_{*} \leq v, \quad w \leq w^{*} .
$$

That is, $v_{*}$ and $w^{*}$ are a pair of minimal-maximal quasisolutions of $(1)$ in $\left[v^{(0)}, w^{(0)}\right]$.

(iii) Since $v_{*}(t) \leq w^{*}(t), t \in[0,1]$, it is sufficient to prove that $v_{*}(t) \geq w^{*}(t), t \in(0,1)$. In fact, from (40) and supposition (iii), we have (notice that $v_{*} \leq w^{*}$ )

$$
\begin{aligned}
& D^{\mu}\left(v_{*}\right.\left.-w^{*}\right) \\
&= f_{0}\left(t, w^{*}\right)-f_{0}\left(t, v_{*}\right)+f_{1}\left(t, w^{*}\right) \\
&-f_{1}\left(t, v_{*}\right)+f_{2}\left(t, v_{*}\right)-f_{2}\left(t, w^{*}\right) \\
& \leq-L_{0}\left(w^{*}-v_{*}\right)+L_{1}\left(w^{*}-v_{*}\right)+L_{2}\left(w^{*}-v_{*}\right) \\
&=-\left(L_{0}-L_{1}-L_{2}\right)\left(w^{*}-v_{*}\right), \\
& v_{*}(0)-w^{*}(0)=0, \quad v_{*}(1)-w^{*}(1)=0 .
\end{aligned}
$$

Thus, by setting $p(t)=v_{*}-w^{*}$, from (46), we obtain

$$
\begin{gathered}
D^{\mu} p(t)-\left(L_{0}-L_{1}-L_{2}\right) p(t) \leq 0 \\
p(0), p(1)=0 .
\end{gathered}
$$

By Lemma 2 (notice that $L_{0}-L_{1}-L_{2}>0$ ), we have that

$$
p(t) \geq 0, \quad t \in[0,1]
$$

Therefore, $v_{*} \geq w^{*}, t \in[0,1]$. Hence $v_{*}=w^{*}$ is the actual solution of (1) in $\left[v^{(0)}, w^{(0)}\right]$. Thus, we complete this proof.

\section{Numerical Implementation}

In this section, the numerical procedures are introduced to obtain an approximate solution of (1).

For the problem (25), from Lemma 3, we have

$$
\begin{aligned}
& v^{(k)}(t) \\
& \qquad \alpha+(\beta-\alpha) t \\
& \qquad-\int_{0}^{1} G(s, t)\left[M v^{(k)}(s)-M v^{(k-1)}(s)\right. \\
& \quad-f_{0}\left(s, v^{(k-1)}(s)\right)-f_{1}\left(s, v^{(k-1)(s)}\right) \\
& \left.\quad-f_{2}\left(s, w^{(k-1)}(s)\right)\right] d s,
\end{aligned}
$$

$$
\begin{aligned}
& w^{(k)}(t) \\
& =\alpha+(\beta-\alpha) t \\
& \quad-\int_{0}^{1} G(s, t)\left[M w^{(k)}(s)-M w^{(k-1)}(s)\right. \\
& \quad-f_{0}\left(s, w^{(k-1)}(s)\right)-f_{1}\left(s, w^{(k-1)}(s)\right) \\
& \left.\quad-f_{2}\left(s, v^{(k-1)}(s)\right)\right] d s .
\end{aligned}
$$

Thus by Theorem 6, the unique solution to problem (1) is given by

$$
\lim _{k \rightarrow \infty} v^{(k)}(t)=v_{*}(t)=w^{*}(t)=\lim _{k \rightarrow \infty} w^{(k)}(t) .
$$


Setting

$$
\begin{aligned}
\Phi(s, t)=G(s, t)[ & M v^{(k)}(s)-M v^{(k-1)}(s) \\
& -f_{0}\left(s, v^{(k-1)}(s)\right)-f_{1}\left(s, v^{(k-1)(s)}\right) \\
& \left.-f_{2}\left(s, w^{(k-1)}(s)\right)\right], \\
\Psi(s, t)=G(s, t)[ & M w^{(k)}(s)-M w^{(k-1)}(s) \\
& -f_{0}\left(s, w^{(k-1)}(s)\right)-f_{1}\left(s, w^{(k-1)}(s)\right) \\
& \left.-f_{2}\left(s, v^{(k-1)}(s)\right)\right] .
\end{aligned}
$$

Discretize the interval $[0,1]$ with the nodes $h=1 / N, t_{i}=i h$, $s_{j}=j h$. Using Simpson's quadrature rule, we can obtain the function $S_{\Phi}(t)$, and $\Psi(t)$, to approximate the integral $\int_{0}^{1} \Phi(s, t) d s$ and $\int_{0}^{1} \Psi(s, t) d s$ on the right hand sid of (49), where

$$
\begin{aligned}
& S_{\Phi}(t)=\frac{h}{3} \sum_{j=0}^{N} d_{j} \Phi\left(s_{j}, t\right), \\
& S_{\Psi}(t)=\frac{h}{3} \sum_{j=0}^{N} d_{j} \Psi\left(s_{j}, t\right),
\end{aligned}
$$

where $\left\{d_{j}\right\}$ are the coefficients in composite Simpson's quadrature rule: $d_{0}=d_{N}=1, d_{2 j-1}=4, d_{2 j}=2,1 \leq j \leq$ $N / 2$.

Now, let $v_{i}^{(k)} \approx v^{(k)}\left(t_{i}\right), w_{i}^{(k)} \approx w^{(k)}\left(t_{i}\right)$, from (49) and the above; we obtain the following linear system of equations mentioned

$$
\begin{aligned}
v_{i}^{(k)}= & v^{(k)}\left(t_{i}\right) \\
= & \alpha+(\beta-\alpha) t_{i}-S_{\Phi}\left(t_{i}\right) \\
= & \alpha+(\beta-\alpha) t_{i}-\frac{h}{3} \sum_{j=0}^{N} d_{j} \Phi\left(s_{j}, t_{i}\right) \\
= & \alpha+(\beta-\alpha) t_{i}-\frac{h}{3} \sum_{j=0}^{N} d_{j} G\left(s_{j}, t_{i}\right) M v_{j}^{(k)} \\
& +\frac{h}{3} \sum_{j=0}^{N} d_{j} G\left(s_{j}, t_{i}\right) \\
& \times\left[M v_{j}^{(k-1)}+f_{0}\left(s_{j}, v_{j}^{(k-1)}\right)+f_{1}\left(s_{j}, v_{j}^{(k-1)}\right)\right] \\
& +\frac{h}{3} \sum_{j=0}^{N} d_{j} G\left(s_{j}, t_{i}\right) f_{2}\left(s_{j}, w_{j}^{(k-1)}\right),
\end{aligned}
$$

$$
\begin{aligned}
w_{i}^{(k)}= & w^{(k)}\left(t_{i}\right) \\
= & \alpha+(\beta-\alpha) t_{i}-S_{\Psi}\left(t_{i}\right) \\
= & \alpha+(\beta-\alpha) t_{i}-\frac{h}{3} \sum_{j=0}^{N} d_{j} \Psi\left(s_{j}, t_{i}\right) \\
= & \alpha+(\beta-\alpha) t_{i}-\frac{h}{3} \sum_{j=0}^{N} d_{j} G\left(s_{j}, t_{i}\right) M w_{j}^{(k)} \\
& +\frac{h}{3} \sum_{j=0}^{N} d_{j} G\left(s_{j}, t_{i}\right) \\
& \times\left[M w_{j}^{(k-1)}+f_{0}\left(s_{j}, w_{j}^{(k-1)}\right)+f_{1}\left(s_{j}, w_{j}^{(k-1)}\right)\right] \\
& +\frac{h}{3} \sum_{j=0}^{N} d_{j} G\left(s_{j}, t_{i}\right) f_{2}\left(s_{j}, v_{j}^{(k-1)}\right) .
\end{aligned}
$$

or

$$
\begin{aligned}
v_{i}^{(k)}+ & \frac{h}{3} \sum_{j=0}^{N} d_{j} G\left(s_{j}, t_{i}\right) M v_{j}^{(k)} \\
= & \alpha+(\beta-\alpha) t_{i}+\frac{h}{3} \sum_{j=0}^{N} d_{j} G\left(s_{j}, t_{i}\right) \\
& \times\left[M v_{j}^{(k-1)}+f_{0}\left(s_{j}, v_{j}^{(k-1)}\right)+f_{1}\left(s_{j}, v_{j}^{(k-1)}\right)\right] \\
& +\frac{h}{3} \sum_{j=0}^{N} d_{j} G\left(s_{j}, t_{i}\right) f_{2}\left(s_{j}, w_{j}^{(k-1)}\right), \\
w_{i}^{(k)}+ & \frac{h}{3} \sum_{j=0}^{N} d_{j} G\left(s_{j}, t_{i}\right) M w_{j}^{(k)} \\
= & \alpha+(\beta-\alpha) t_{i}+\frac{h}{3} \sum_{j=0}^{N} d_{j} G\left(s_{j}, t_{i}\right) \\
& \times\left[M w_{j}^{(k-1)}+f_{0}\left(s_{j}, w_{j}^{(k-1)}\right)+f_{1}\left(s_{j}, w_{j}^{(k-1)}\right)\right] \\
& +\frac{h}{3} \sum_{j=0}^{N} d_{j} G\left(s_{j}, t_{i}\right) f_{2}\left(s_{j}, v_{j}^{(k-1)}\right) .
\end{aligned}
$$

Setting

$$
\begin{aligned}
& G_{i j}=\frac{h}{3} d_{j} G\left(s_{j}, t_{i}\right), \quad \mathscr{G}=\left(G_{i j}\right)_{(N+1) \times(N+1)} \\
& \mathscr{A}_{i}\left(v^{(k-1)}\right) \\
& =\alpha+(\beta-\alpha) t_{i} \\
& +\sum_{j=0}^{N} G_{i j}\left[M v_{j}^{(k-1)}+f_{0}\left(s_{j}, v_{j}^{(k-1)}\right)+f_{1}\left(s_{j}, v_{j}^{(k-1)}\right)\right],
\end{aligned}
$$




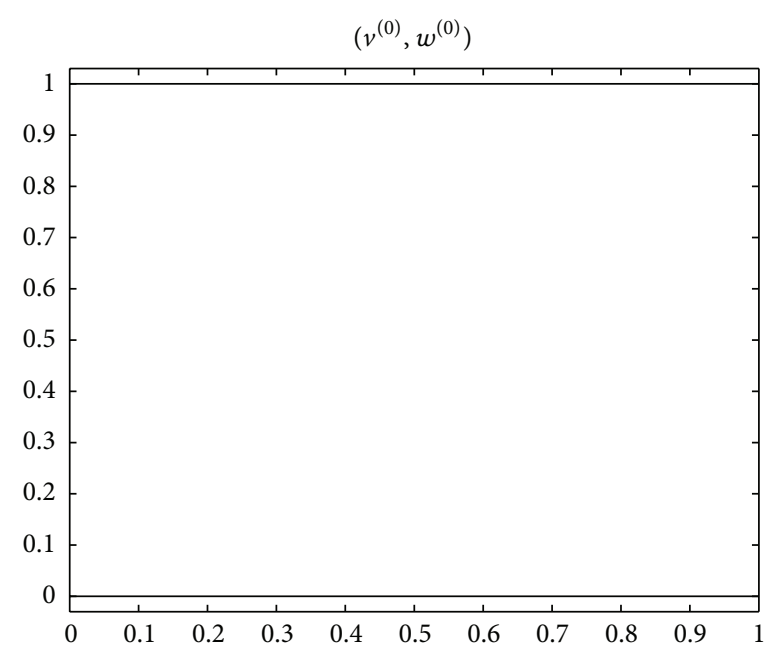

(a)

$\left(v^{(2)}, w^{(2)}\right)$

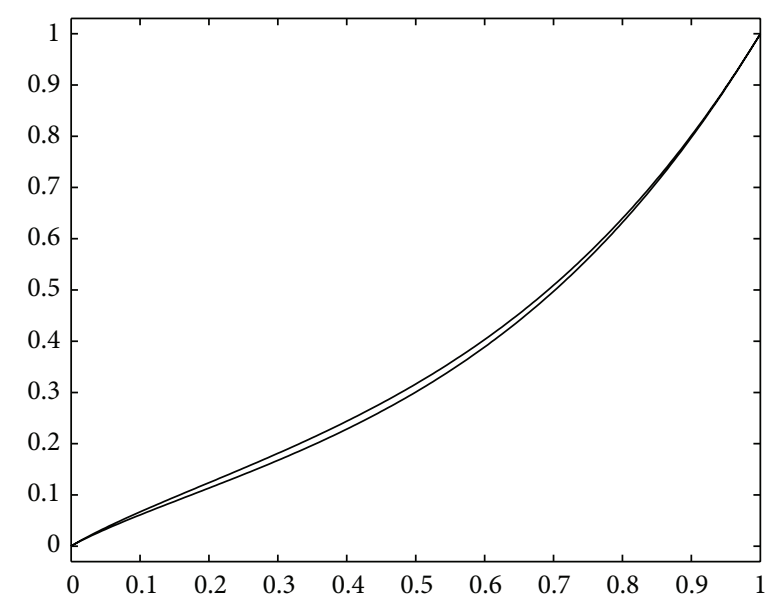

(c)

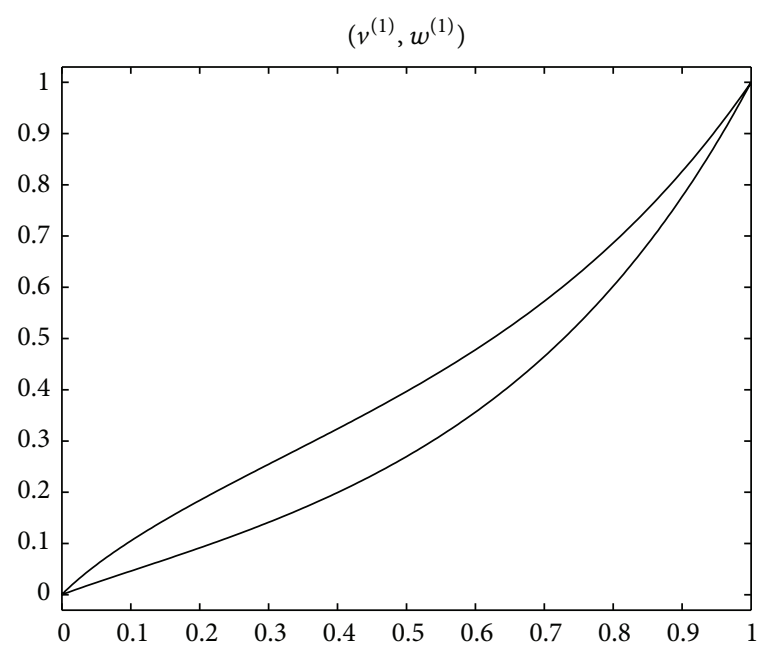

(b)

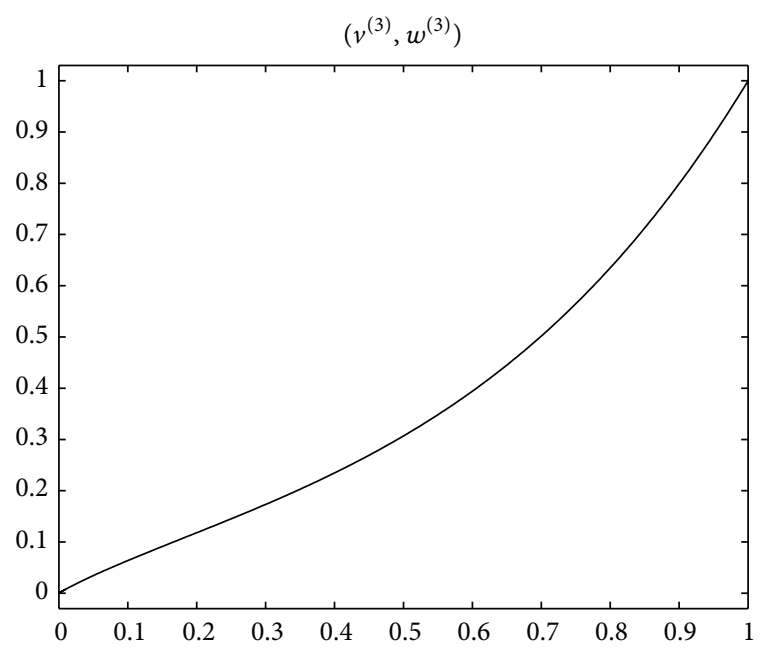

(d)

Figure 1: Graph of $v^{(k)}$ and $w^{(k)}$.

$$
\begin{aligned}
& \mathscr{B}_{i}\left(w^{(k-1)}\right) \\
& =\alpha+(\beta-\alpha) t_{i} \\
& \quad+\sum_{j=0}^{N} G_{i j}\left[M w_{j}^{(k-1)}+f_{0}\left(s_{j}, w_{j}^{(k-1)}\right)+f_{1}\left(s_{j}, w_{j}^{(k-1)}\right)\right], \\
& \mathscr{V}^{(k)}=\left(v_{0}^{(k)}, v_{1}^{(k)}, \ldots, v_{N}^{(k)}\right)^{T}, \\
& \quad \mathscr{W}^{(k)}=\left(w_{0}^{(k)}, w_{1}^{(k)}, \ldots, w_{N}^{(k)}\right)^{T}, \\
& \quad \mathscr{A}\left(v^{(k-1)}\right) \\
& =\left(\mathscr{A}_{0}\left(v^{(k-1)}\right), \mathscr{A}_{1}\left(v^{(k-1)}\right), \ldots, \mathscr{A}_{N}\left(v^{(k-1)}\right)\right)^{T}, \\
& \mathscr{B}\left(w^{(k-1)}\right) \\
& =\left(\mathscr{B}_{0}\left(w^{(k-1)}\right), \mathscr{B}_{1}\left(w^{(k-1)}\right), \ldots, \mathscr{B}_{N}\left(w^{(k-1)}\right)\right)^{T},
\end{aligned}
$$

$$
\begin{aligned}
& \mathscr{P}\left(w^{(k-1)}\right) \\
& \quad=\left(f_{2}\left(s_{0}, w_{0}^{(k-1)}\right), f_{2}\left(s_{1}, w_{1}^{(k-1)}\right), \ldots, f_{2}\left(s_{N}, w_{N}^{(k-1)}\right)\right)^{T}, \\
& \mathscr{Q}\left(v^{(k-1)}\right) \\
& \quad=\left(f_{2}\left(s_{0}, v_{0}^{(k-1)}\right), f_{2}\left(s_{1}, v_{1}^{(k-1)}\right), \ldots, f_{2}\left(s_{N}, v_{N}^{(k-1)}\right)\right)^{T} .
\end{aligned}
$$

Thus system (54) can be written as a matrix-vector equation

$$
\begin{aligned}
& (\mathscr{I}+M \mathscr{G}) \mathscr{V}^{(k)}=\mathscr{A}\left(v^{(k-1)}\right)+\mathscr{G P}\left(w^{(k-1)}\right), \\
& (\mathscr{I}+M \mathscr{G}) \mathscr{W}^{(k)}=\mathscr{B}\left(w^{(k-1)}\right)+\mathscr{G} \mathscr{Q}\left(v^{(k-1)}\right),
\end{aligned}
$$


TABLE $1: E(k): k=2 i, i=0,1,2,3,4,5$.

\begin{tabular}{lcccccc}
\hline$k$ & 0 & 2 & 4 & 6 & 8 & 10 \\
\hline$E(k)$ & 1.000 & $1.117 \times 10^{-2}$ & $1.302 \times 10^{-4}$ & $1.259 \times 10^{-6}$ & $1.048 \times 10^{-8}$ & $7.632 \times 10^{-11}$ \\
\hline
\end{tabular}

Remark 7. Applying the above numerical scheme, we can solve iteratively for $v^{(k)}$ and $w^{(k)}$ up to some large enough $k=K$. For a given accuracy $\varepsilon$, we take $v^{(k)}$ and $w^{(k)}$ as $\varepsilon$ accurate approximations of $v_{*}$ and $w^{*}$, respectively. And we use

$$
E(k)=\left\|w^{(k)}-v^{(k)}\right\|_{2}=\left(\int_{0}^{1}\left(w^{(k)}(t)-v^{(k)}(t)\right)^{2} d t\right)^{1 / 2}
$$

to describe the error.

Example 8. Consider the fractional BVP

$$
\begin{gathered}
D^{3 / 2} u-4 u+u^{2}+e^{-u}=0, \quad t \in(0,1), \\
u(0)=0, \quad u(1)=1 .
\end{gathered}
$$

Let

$$
\begin{gathered}
\mu=\frac{3}{2}, \quad \alpha=0, \quad \beta=1, \quad f_{0}(t, u)=-4 u, \\
f_{1}(t, u)=u^{2}, \quad f_{2}(t, u)=e^{-u} .
\end{gathered}
$$

It can be easily verified that

$$
v^{(0)}(t)=0, \quad w^{(0)}(t)=1
$$

are a pair of ordered quasi-lower and quasi-upper solutions of (58). Moreover,

$$
M=L_{0}=4, \quad L_{1}=2, \quad L_{2}=1, \quad \Lambda=\frac{81}{8} .
$$

Thus

$$
M<\Gamma(\mu) \Lambda, \quad L_{1}+Ł_{2}<L_{0} \leq M .
$$

Hence for this example, we found that, for $\varepsilon=10^{-10}$, it has $K=10$ iterations for $E(k)<\varepsilon$. the graph of $v^{(k)}$ and $w^{(k)}$, for some values of $k$, is in Figure 1 . Table 1 displays $E(k)$ versus $k$ for selected values of $k$.

\section{Acknowledgment}

This work was partially supported by NNSF of China (Grant no. 11171122).

\section{References}

[1] I. Podlubny, Fractional Differential Equations, vol. 198 of Mathematics in Science and Engineering, Academic Press, San Diego, Calif, USA, 1999.

[2] V. Lakshmikantham, S. Leela, and J. Vasundhara, Theory of Fractional Dynamic Systems, Cambridge Academic Publishers, Cambridge, Mass, USA, 2009.
[3] Q. M. Al-Mdallal, "On the numerical solution of fractional Sturm-Liouville problems," International Journal of Computer Mathematics, vol. 87, no. 12, pp. 2837-2845, 2010.

[4] S. Momani and Z. Odibat, "Analytical approach to linear fractional partial differential equations arising in fluid mechanics," Physics Letters A, vol. 355, pp. 271-279, 2006.

[5] S. Momani and N. Shawagfeh, "Decomposition method for solving fractional Riccati differential equations," Applied Mathematics and Computation, vol. 182, no. 2, pp. 1083-1092, 2006.

[6] Q. M. Al-Mdallal, M. I. Syam, and M. N. Anwar, "A collocationshooting method for solving fractional boundary value problems," Communications in Nonlinear Science and Numerical Simulation, vol. 15, no. 12, pp. 3814-3822, 2010.

[7] F. Jiao and Y. Zhou, "Existence results for fractional boundary value problem via critical point theory," International Journal of Bifurcation and Chaos, vol. 22, no. 4, Article ID 1250086, 2012.

[8] F. Jiao and Y. Zhou, "Existence of solutions for a class of fractional boundary value problems via critical point theory," Computers \& Mathematics with Applications, vol. 62, no. 3, pp. 1181-1199, 2011.

[9] C. F. Li, X. N. Luo, and Y. Zhou, "Existence of positive solutions of the boundary value problem for nonlinear fractional differential equations," Computers \& Mathematics with Applications, vol. 59, no. 3, pp. 1363-1375, 2010.

[10] S. Abbas and M. Benchohra, "Upper and lower solutions method for impulsive partial hyperbolic differential equations with fractional order," Nonlinear Analysis: Hybrid Systems, vol. 4, no. 3, pp. 406-413, 2010.

[11] M. Benchohra and S. Hamani, "The method of upper and lower solutions and impulsive fractional differential inclusions," Nonlinear Analysis: Hybrid Systems, vol. 3, no. 4, pp. 433-440, 2009.

[12] A. Shi and S. Zhang, "Upper and lower solutions method and a fractional differential equation boundary value problem," Electronic Journal of Qualitative Theory of Differential Equations, vol. 30, pp. 1-13, 2009.

[13] G. Wang, "Monotone iterative technique for boundary value problems of a nonlinear fractional differential equation with deviating arguments," Journal of Computational and Applied Mathematics, vol. 236, no. 9, pp. 2425-2430, 2012.

[14] M. Al-Refai and M. Ali Hajji, "Monotone iterative sequences for nonlinear boundary value problems of fractional order," Nonlinear Analysis: Theory, Methods \& Applications, vol. 74, no. 11, pp. 3531-3539, 2011.

[15] H. L. Royden, Real Analysis, Macmillan Publishing Company, New York, NY, USA, 3rd edition, 1988. 


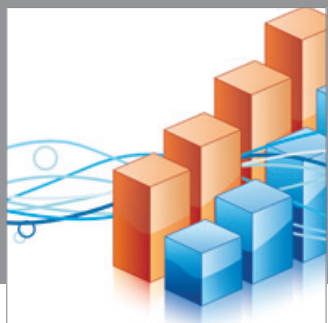

Advances in

Operations Research

mansans

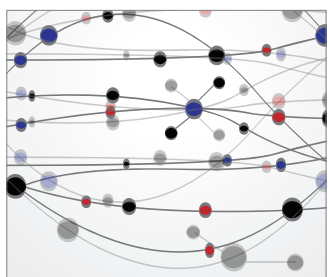

The Scientific World Journal
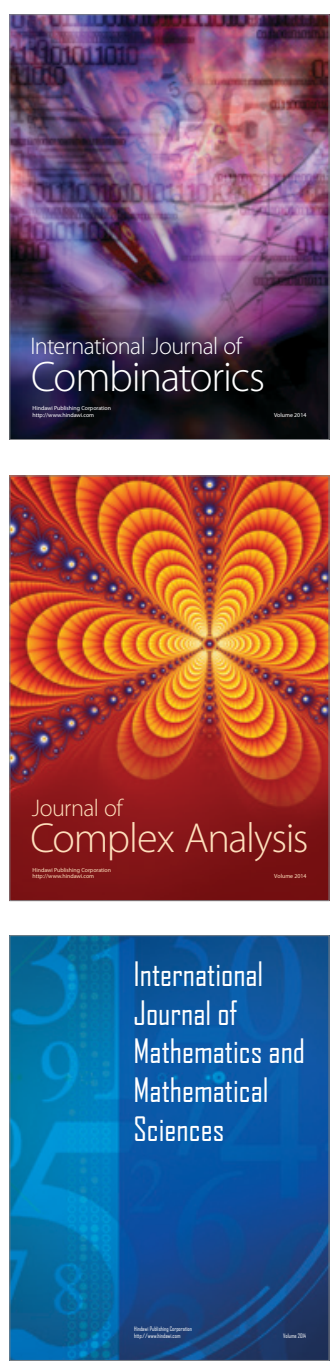
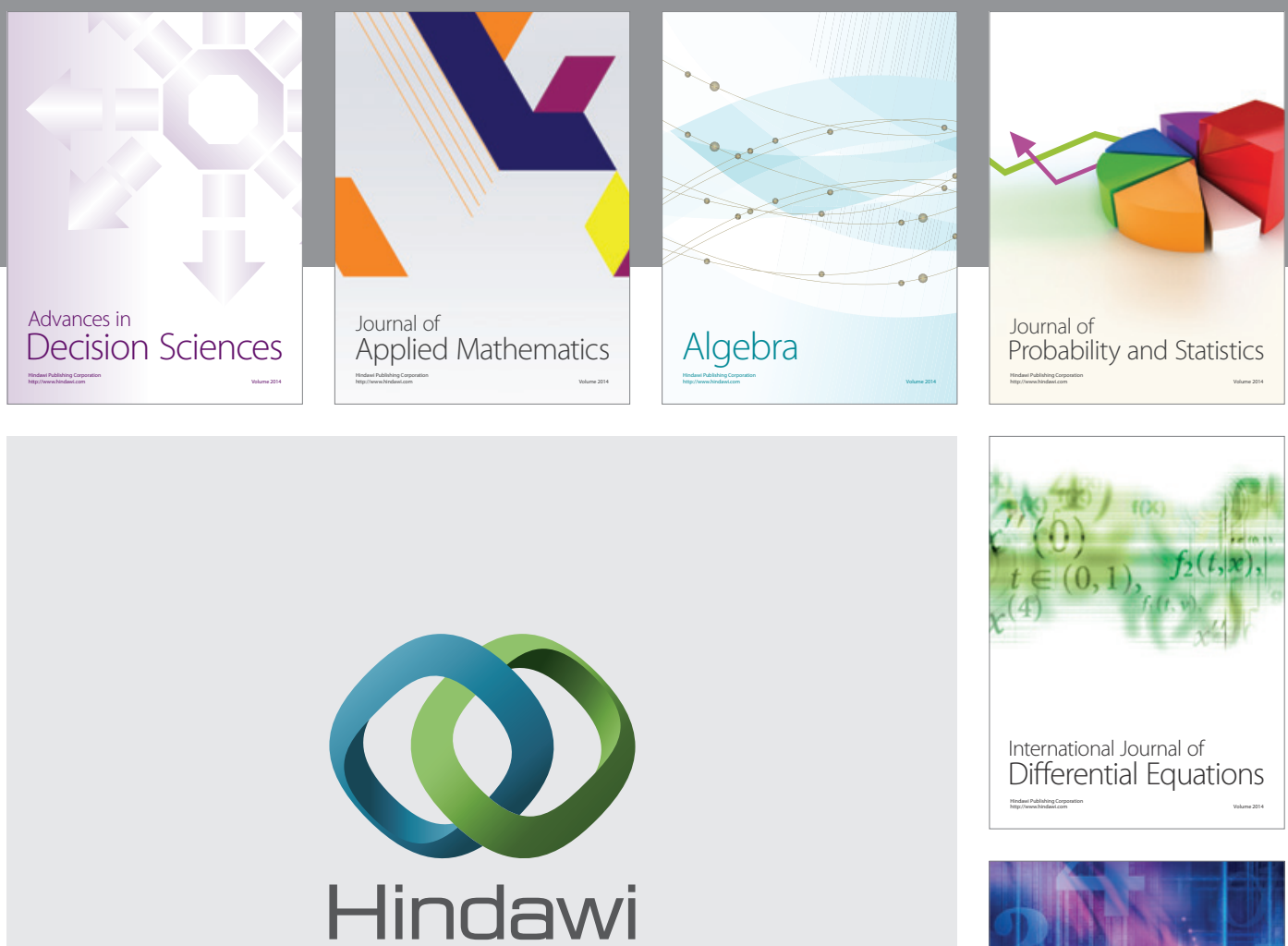

Submit your manuscripts at http://www.hindawi.com
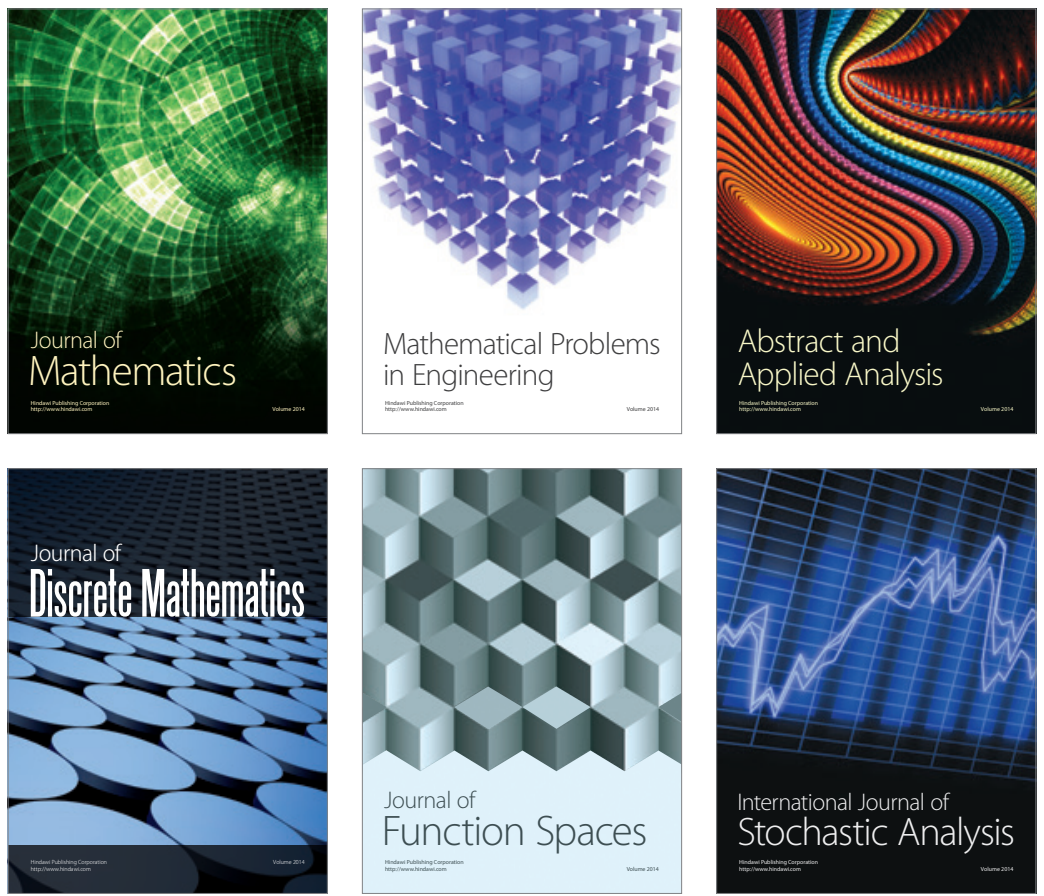

Journal of

Function Spaces

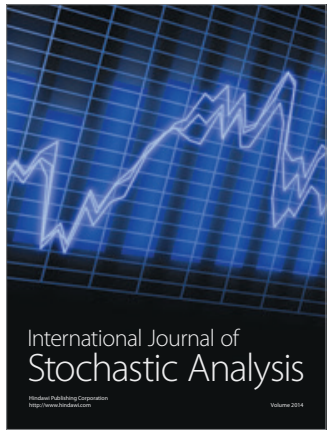

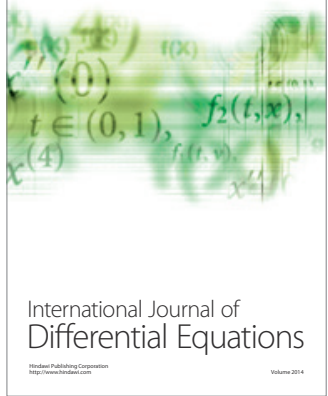
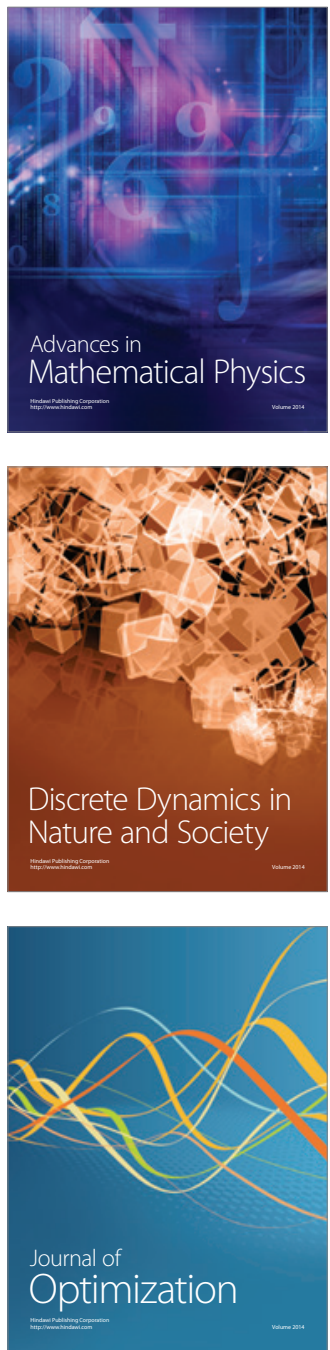